\title{
ON CERTAIN TYPES OF SOLUTION OF THE EQUATION OF HEAT CONDUCTION
}

\author{
$b y$ STEWART PATERSON \\ (Received 20th December, 1949)
}

Let $f(x, y, z, t)$ satisfy the equation

$$
f_{t}=\nabla^{2} f=f_{x x}+f_{y y}+f_{z x}
$$

For certain purposes, particularly in connection with the propagation of a boundary of fusion etc., it is of interest to discover solutions of (1) which permit the equation :

$$
f=\text { constant, }
$$

to be solved explicitly in the form :

$$
g(x, y, z)=h(t)
$$

This suggests the examination of solutions of the type

$$
f=f(\zeta)
$$

where

$$
\zeta \equiv \phi(x, y, z) \cdot \psi(t) \text {, }
$$

and $f, \phi, \psi$ are functions to be determined. To save repetition, Roman capitals denote arbitrary constants throughout.

1. Linear system. $f=f(x, t)=f[\zeta(x, t)] ; f_{t}=f_{x x}$.

(a) Let $\zeta \equiv x^{m} t^{n}$. Then, by (1),

That is,

$$
n x^{2} f^{\prime}(\zeta)=m(m-1) t f^{\prime}(\zeta)+m^{2} x^{m} t^{n+1} f^{\prime \prime}(\zeta)
$$

$$
\frac{f^{\prime \prime}}{f^{\prime}}=\frac{n x^{2+\frac{m}{n}}-m(m-1) \zeta^{\frac{1}{n}}}{m^{2} \zeta^{1+\frac{1}{n}}} .
$$

Consequently

$$
n=0, \text { or } m=-2 n \text {. }
$$

If $m=-2 n \neq 0$, we may without loss of generality take $n=-\frac{1}{2}$, whereupon $m=1$, and

$$
f^{\prime \prime} \mid f^{\prime}=-\zeta / 2 \text {. }
$$

Then

$$
f^{\prime}=A e^{-\zeta^{2} / 4} \text {, }
$$

and so

$$
f(\zeta)=B \operatorname{erf}(\zeta / 2)+C
$$

$n=0$ yields the trivial case

$$
f(\zeta)=A \zeta^{\frac{1}{m}}+B=A x+B
$$

which may be regarded as a limiting form of $(10)$.

The only solutions of (1) of the form $f\left(x^{m} t^{n}\right)$ are therefore

$$
\begin{gathered}
f=B \operatorname{erf} *\left(\frac{x}{2 \sqrt{t}}\right)+C, \\
f=A x+B . \ldots \ldots \ldots . \\
* \operatorname{erf}(x) \equiv \frac{2}{\sqrt{\pi}} \int_{0}^{x} e^{-u^{2}} d u .
\end{gathered}
$$


(b) To generalise, let now $\zeta \equiv \phi(x) \psi(t)$.

Here

$$
f_{t}=\phi \dot{\psi} f^{\prime} \text { and } f_{x x}=\ddot{\phi} \psi f^{\prime}+\dot{\phi}^{2} \psi^{2} f^{\prime \prime},
$$

where the dot signifies differentiation with respect to $x$ or $t$. Consequently, by (1),

If we denote $f^{\prime \prime} \mid f^{\prime}$ by $\theta(\zeta)$, then

$$
f^{\prime \prime} \mid f^{\prime}=\frac{\phi \dot{\psi}-\psi \ddot{\phi}}{\dot{\phi}^{2} \psi^{2}}
$$

$$
\theta_{x}=\theta^{\prime} \psi \phi \quad \text { and } \quad \theta_{t}=\theta^{\prime} \phi \psi
$$

Consequently

$$
\phi \psi \theta_{x}=\psi \phi \theta_{t},
$$

and from (14) and (15), after some reduction, we have

which is of the form :

$$
\frac{2 \phi \dddot{\phi}}{\dot{\phi}^{2}} \cdot \frac{\dot{\psi}}{\psi}=\left(\frac{3 \psi}{\psi}-\frac{\ddot{\psi}}{\dot{\psi}}\right)+\left(\frac{2 \ddot{\phi}^{2}}{\dot{\phi}^{2}}-\frac{\dddot{\phi}}{\dot{\phi}}-\frac{\ddot{\phi}}{\phi}\right),
$$

$$
f_{1}(x) f_{2}(t)=f_{3}(t)+f_{4}(x) .
$$

It follows that either $f_{1}$ or $f_{2}$ is a constant. That is,

In the former case,

$$
\phi \ddot{\phi}=B \dot{\phi}^{2} \quad \text { or } \quad \dot{\psi}=A^{2} \psi
$$

so that $f_{4}(x) \equiv 0$. Also

$$
\phi \dddot{\phi}+\dot{\phi} \ddot{\phi}=2 B \dot{\phi} \ddot{\phi}=2 \phi \ddot{\phi}^{2} / \phi,
$$

$$
\phi=C \phi^{B}, \quad \phi^{1-B}=(1-B)(C x+D) .
$$

and we lose no generality in setting $B=0$, so that

Then

$$
\phi=C x+D \text {. }
$$

whence

$$
3 \dot{\psi} / \psi=\ddot{\psi} / \dot{\psi}
$$

$$
\dot{\psi}=-E \psi^{3}, \quad \psi=1 / 2 \sqrt{ }(E t+F)
$$

and we may, without loss, take $E=2 C^{2}$.

Finally, by (14), (18) and (21),

$$
f^{\prime \prime} / f^{\prime}=-\frac{E \zeta}{C^{2}}=-2 \zeta
$$

which leads to

$$
f(\zeta)=P \operatorname{erf}(\zeta)+Q
$$

Thus, when $\phi \ddot{\phi} / \phi^{2}=$ constant, the only solution is

$$
\begin{aligned}
& f=P \operatorname{erf}\left(\frac{x+R}{2 \sqrt{t+S}}\right)+Q . \\
& f=P x+Q \quad \ldots \ldots \ldots \ldots \ldots
\end{aligned}
$$

is again a trivial limiting case.

$$
\text { If } \dot{\psi}=A^{2} \psi \text {, }
$$

$$
f_{3}(t)=2 A^{2} \text { and } \quad \psi=B e^{A^{2} t}
$$


Also, by (14),

so that

$$
f^{\prime \prime} \mid f^{\prime}=\frac{A^{2} \phi-\ddot{\phi}}{\dot{\phi}^{2}} \cdot \frac{\phi}{\zeta},
$$

$$
\dddot{\phi}-A^{2} \phi=C \dot{\phi}^{2} / \phi
$$

The term $C \dot{\phi}^{2} / \phi$ can be removed by setting $\phi \equiv \Phi^{\frac{1}{1-C}}$, so that we may take $C=0$, giving

Then, by (27),

$$
\phi=D e^{A x}+E e^{-A x} \text {. }
$$

which implies

$$
f^{\prime \prime} \mid f^{\prime}=0 \text {, }
$$

$$
f=F \zeta+G \text {. }
$$

Collecting (26), (28), and (30), we conclude that when $\psi / \psi=$ constant, the only solution is

$$
f=e^{A^{2} t}\left[P e^{A x}+Q e^{-A x}\right]+R \text {. }
$$

$A$ may be imaginary, in which case $P$ and $Q$ are complex.

The only solutions $f[\phi(x), \psi(t)]$ of $f_{t}=f_{x x}$ are therefore : (24), (25) and (31).

2. Cylindrical system $f=f(r, t)=f[\zeta(r, t)] ; f_{t}=f_{r r}+\frac{1}{r} f_{r}$.

Let $\zeta \equiv \phi(r) \psi(t)$.

Then

$$
\theta(\zeta) \equiv f^{\prime \prime} \mid f^{\prime}=\frac{\phi \dot{\psi}-\ddot{\phi} \psi-\frac{1}{r} \dot{\phi} \psi}{\dot{\phi}^{2} \psi^{2}}
$$

and (15) leads to

$$
\frac{2 \phi \ddot{\phi}}{\dot{\phi}^{2}} \cdot \frac{\dot{\psi}}{\psi}=\left(\frac{3 \dot{\psi}}{\psi}-\frac{\ddot{\psi}}{\dot{\psi}}\right)+\left(\frac{2 \dddot{\phi}^{2}}{\dot{\phi}^{2}}-\frac{\dddot{\phi}}{\phi}-\frac{\ddot{\phi}}{\phi}+\frac{1}{r^{2}}+\frac{1}{r} \frac{\ddot{\phi}}{\dot{\phi}}-\frac{1}{r} \frac{\dot{\phi}}{\phi}\right),
$$

whence

$$
\phi \ddot{\phi}=B \dot{\phi}^{2} \quad \text { or } \quad \dot{\psi}=-A^{2} \psi .
$$
upon

In the former case, $\dot{\phi}=C \phi^{B}, \phi^{1-B}=C(1-B)(r+D)$, and we may take $B=0, C=1$, where-

Then, by (33)

$$
\ddot{\phi}=0, \quad \dot{\phi}=1, \quad \phi=r+D .
$$

so that $D=0$, giving $\phi=r$. Also

$$
0=\left(\frac{3 \dot{\psi}}{\psi}-\frac{\ddot{\psi}}{\dot{\psi}}\right)+\frac{1}{r^{2}}-\frac{1}{r(r+D)},
$$

$$
\psi \ddot{\psi}=3 \dot{\psi}^{2}, \quad \dot{\psi}=-E \psi^{3}, \quad \psi=1 / \sqrt{2 E(t+R)} .
$$

We may take $E=2$, whence

Finally, by (32),

$$
\zeta=\frac{r}{2 \sqrt{t+R}}
$$

which leads to *

$$
f^{\prime \prime} \mid f^{\prime}=-2 \zeta-\frac{1}{\zeta}
$$

$$
\begin{gathered}
f=P E i\left(\frac{-r^{2}}{4(t+R)}\right)+Q . \\
* E i(x) \equiv \int_{\infty}^{-\infty} e^{-u} d u / u
\end{gathered}
$$


Again, if $=\psi-A^{2} \psi, \psi=B e^{-A^{2} t}$ and

$$
f^{\prime \prime} \mid f^{\prime}=-\frac{\phi}{\zeta} \cdot \frac{A^{2} \phi+\ddot{\phi}+\dot{\phi} / r}{\dot{\phi}^{2}}
$$

30 that

$$
\ddot{\phi}+\frac{1}{r} \dot{\phi}+A^{2} \phi=C \dot{\phi}^{2} / \phi
$$

The term, $C \dot{\phi}^{2} / \phi$ can be removed by substituting $\phi=\Phi^{\frac{1}{1-C}}$, so that we may without loss iake $C=0$. The solution of (39) is then

$$
\phi=D J_{0}(A r)+E Y_{0}(A r),
$$

where $J_{0}$ and $Y_{0}$ are zero-order Bessel functions of first and second kinds respectively.

Finally, by (38),

o that

$$
f^{\prime \prime} / f^{\prime}=0
$$

Jollecting the results,

$$
f=G \zeta+R
$$

$$
f=e^{-A^{2} t}\left[P J_{0}(A r)+Q Y_{0}(A r)\right]+R .
$$

1 may again be imaginary, with $P, Q$ complex.

When $A=0$, we have the trivial limiting case

$$
f=P \ln r+Q \text {. }
$$

$37),(42)$ and (43) represent the only solutions $f[\phi(r) . \psi(t)]$ of $f_{t}=f_{r r}+\frac{1}{r} f_{r}$.

3. Spherical system $f=f(r, t)=f[\zeta(r, t)] ; f_{t}=f_{r r}+\frac{2}{r} f_{r}$. As before, let $\zeta \equiv \phi(r) \psi(t)$. The ylindrical solution applies, except that $1 / r$ must be replaced by $2 / r$ in (32) and (33). (35) fol. Jws as before, but (36) becomes

$$
f^{\prime \prime} \mid f^{\prime}=-2 \zeta-\frac{2}{\zeta}
$$

rhich leads to

$$
f=P\left[\frac{\sqrt{t+R}}{r} e^{-\frac{r^{2}}{4(t+R)}}+\frac{\sqrt{\pi}}{2} \operatorname{erf} \frac{r}{2 \sqrt{t+R}}\right]+Q
$$

Again, if $\psi=A^{2} \psi,(39)$ becomes

$$
\ddot{\phi}+\frac{2}{r} \dot{\phi}-A^{2} \phi=C \dot{\phi}^{2} / \phi
$$

nd the term $C \dot{\phi}^{2} / \phi$ may be removed as before, so that we take $C=0$, whereupon

$$
\phi=\left[D e^{A r}+E e^{-A r}\right] / r .
$$

(41) still applies, and so

$$
f=\frac{e^{A 2 t}}{r}\left[P e^{A r}+Q e^{-A r}\right]+R
$$

! may again be imaginary, with complex $P, Q$. 
When $A=0$, we have the trivial limiting case

$$
f=\frac{P}{r}+Q \text {. }
$$

(45), (48) and (49) are the only solutions

$$
f[\phi(r) \cdot \psi(t)] \text { of } f_{t}=f_{r r}+\frac{2}{r} f_{r}
$$

Imperial Chemical Industries LTd

Nobel Division Research Department

Stevenston 